\title{
Wie feldabhängig sind Coaching und Supervision?
}

\section{Astrid Schreyögg}

Die obige Frage lässt sich natürlich auch umkehren: „Wie feld-un-abhängig sind Coaching und Supervision“. Mancher wird sich jetzt fragen, warum sollte das jeweilige Feld bei der Anwendung eines Beratungsformats überhaupt eine Rolle spielen? Kühl (2008) hat die bemerkenswerte Feststellung gemacht, dass es sich bei der Sozialarbeit um das „Wirtstier“ der Supervision handelt. Ich stelle mir also ein gutmütiges, Nashorn-ähnliches Tier vor, auf dessen Rücken bunte „Supervisionsvögel“" sitzen und ihre Nahrung genussvoll herauspicken. Mit diesem Bild lässt sich veranschaulichen, dass ein jeweiliges Format niemals total losgelöst von seinen Ursprüngen und seiner Entstehungsgeschichte zu denken ist. Und tatsächlich sind die gesamte Entwicklung von Supervision (Belardi 1992), ihre konzeptionelle Einbettung wie auch ihre Praxis stark an die Sozialarbeit mit ihren Ausbildungsstätten gekoppelt. So ist Supervision bis heute besonders markant in den Bereichen von Sozialarbeit und Sozialpädagogik verankert. Daraus folgte im Weiteren eine Präferenz für Felder, die der Sozialarbeit nahestehen wie die Psychotherapie und die Pädagogik. Damit bleibt aber immer die Frage prekär, wie gut sich Supervision in Feldern verankern kann, die von der Sozialarbeit weiter entfernt sind. So ist es für Supervisoren bislang ziemlich mühsam, in der Wirtschaft Fuß zu fassen, zumal der Begriff „Supervision“ hier oft mit seiner Variante, der „Administrative Supervision“, vermischt wird. Der Hinweis, dass es sich bei dem angebotenen Format ja um „Clinical Supervision" handelt, führt meistens erst recht zu Missverständnissen, weil sich potenzielle Supervisanden dann nämlich unangemessen klientifiziert fühlen.

Wer oder was ist aber nun eigentlich der Wirt von Coaching? Manche meinen, dass der Sportbereich maßgeblich war, weil in der Coaching-Szene immer wieder ehedem erfolgreiche Tennis- oder Golftrainer wie etwa Whitmore (2009) in Erscheinung treten. Böning (2005) behauptet demgegenüber, dass Coaching eher im Dunstkreis von betrieblichen Personalabteilungen entstand. Danach lassen sich als Wirtstiere von Coaching US-amerikanische HR-Protagonisten vermuten. Aber ist das sicher? Im Gegensatz zur Professionsentwicklung von Supervision befindet sich Coaching bislang in einem viel

\footnotetext{
Online publiziert: 02.08 .2011

(C) VS Verlag für Sozialwissenschaften 2011

Dr. A. Schreyögg $(\square)$

Breisgauer Str. 29, 14129 Berlin, Deutschland

E-Mail: info@schreyoegg.de
} 
diffuseren Stadium im Hinblick auf seine Entstehungsgeschichte, seine Zielsetzungen usw. Fest steht nur, dass es sich um eine Beratungsform handelt, die Menschen mit Steuerungsfunktionen zu mehr Selbstmanagement führen will. Heute ringen immer noch viele Ausbildungsinstitute und viele Verbände um Anerkennung (Kühl 2008). Im Hinblick auf seine Feldoffenheit ist das allerdings durchaus als Vorteil zu werten; denn unter dem Etikett „Führungs-“ bzw. „Managementberatung“ könnte es für die unterschiedlichsten Felder in Frage kommen vom Sport über Unternehmen, die öffentliche Verwaltung, Kliniken bis zu theologischen Milieus. Denn Management- bzw. Strukturierungsfunktionen fallen ja heute in allen diesen Feldern an, und damit wäre auch Coaching potenziell überall zu platzieren.

In den nachfolgenden Beiträgen ist nun zu überprüfen, als wie feldvariabel sich Coaching und Supervision tatsächlich erweisen. Taugt Coaching für die Beratung in Krankenhäusern, in der öffentlichen Verwaltung und in Kontexten, die kirchlich angebunden sind?

Zunächst thematisiert Tobias Felsing, inwieweit Coaching in der öffentlichen Verwaltung bei der Bewältigung von Konflikten dienlich ist. Der Autor bemerkt zunächst, dass Konflikte in Behörden aufgrund der Tatsache, dass Coaching hier noch kaum verbreitet ist, oft schon stark eskaliert sind, bevor einer der „Streithansel“" oder beide ins Konfliktcoaching kommen. Abschließend schildert der Autor aber ein Beispiel, in dem es im Verlauf der Beratung zu seiner eigenen Überraschung am Ende doch noch zu einer Einigung kommt.

Im zweiten Beitrag greift Nils Kollmar das Thema Burnout bei Führungskräften in einer öffentlichen Verwaltung auf mit der Fragestellung, in welcher Weise ein organisationsinterner Coach zu einer Verhaltensänderung beitragen kann. Nach Überlegungen zur Diagnostik, um die Möglichkeiten eines Coachings gegenüber einer Psychotherapie eingrenzen zu können, legt der Autor dar, dass das Format des Einzelcoachings am ehesten erfolgversprechend ist, im Vergleich zu herkömmlichen Ansätzen des Stressmanagements, die oft durch eine Blindheit gegenüber individuellen Erlebensqualitäten geprägt sind. Dies wird anhand von zwei Fallbeispielen exemplifiziert, wobei auch die Besonderheiten des organisationsinternen Coachings erörtert werden.

Was ist aber nun mit Coaching in kirchlichen Kontexten? Supervision hatte sich hier ja schon seit vielen Jahren durchgesetzt. Ja, dieses Format wurde über weite Strecken sogar von den Kirchen in Fortbildung und Praxis stark gefördert. Klaus Götz hat im Rahmen eines Projekts „Kirche coacht Führungskräfte“ bei Führungskräften im Bereich der Erzdiözese Freiburg untersucht, inwieweit Führungskräfte aus Unternehmen sich von Vertretern der Kirche coachen lassen würden. Aus der Befragung ergibt sich zweierlei: Die Führungskräfte befürworten allesamt Coaching, und sie fänden es durchaus passend, von Personen mit kirchlicher Anbindung gecoacht zu werden. Voraussetzung sei aber, dass die Berater etwas von Wirtschaft verstehen. Deshalb sollten Coachingausbildungen auch Managementwissen beinhalten.

Im nachfolgenden Beitrag konfrontiert uns Jutta Polzius mit einer Gruppensupervision, die Menschen aus unterschiedlichen Nationalitäten umfasst. Die Autorin zeigt, dass Supervision, die ja immer sprachlich erfolgt, mit Menschen unterschiedlicher Nationalitäten, die der deutschen Sprache oft nur begrenzt mächtig sind, ganz besondere Anforderungen stellt: Viel Geduld, die Bereitschaft, sich irritieren zu lassen, Ernst-Nehmen des 
Nonverbalen und immer wieder Fragen-Stellen. So zeigen die Beiträge von Götz und von Polzius, dass Coaching versus Supervision je nach dem Arbeitsfeld stark zu modifizieren sind.

Die beiden nachfolgenden Beiträge beziehen sich auf generelle Themen von Coaching: Im ersten präsentiert Andreas Hirschi ein theorie- und methodenbasiertes Modell für das Karriere-Coaching. Er leistet damit einen wertvollen Beitrag für ein sehr häufiges Anliegen im Coaching. Marius Neukom, Kathrin Schnell und Brigitte Boothe haben sich im Rahmen einer empirischen Studie mit der Arbeitsbeziehung im Coaching befasst. Die Autoren kommen nach einer Befragung von Coaching-Verantwortlichen aus Schweizer Firmen zu dem Ergebnis, dass die Fähigkeit des Coachs, eine Beziehung zum Klienten aufzubauen, ganz zentral ist.

Im Praxisbericht geht es noch einmal um Burnout und um die Abgrenzung zwischen Psychotherapie und Coaching: Elisabeth Behrends-Krahnen, Ärztin in einer Klinik für psychosomatische Medizin, zeigt, dass Burnout häufig mit Depressionen verwechselt wird. Anhand von Beispielen postuliert die Autorin aber, dass Burnout durch Coaching zu bessern ist, Depressionen dagegen nicht. So wird deutlich, wie wichtig eine genaue Diagnostik ist, damit die beiden Formate Coaching und Psychotherapie ihre jeweiligen Möglichkeiten in angemessener Weise wahrnehmen können.

Der Diskurs von Stefan Klaussner enthält eine wahrhaft provozierende These: Da Führung ein interaktives Phänomen ist, werden auch miserable Führungsprozesse, die unter dem Stichwort „Abusive Supervision“ beforscht werden, gemeinschaftlich erzeugt, nämlich von der Führungskraft und dem Geführten. Wenn das aber so ist, müssten eigentlich beide gleichzeitig ins Coaching, um ihre Beziehung zum Besseren zu wenden. Na, das sollten wir dann auch in den Firmen durchsetzen, oder?

\section{Literatur}

Belardi, N. (1992). Von der Praxisberatung zur Organisationsentwicklung. Paderborn: Junfermann.

Böning, U. (2005). Coaching: Der Siegeszug eines Personalentwicklungs-Instruments - Eine 15Jahres-Bilanz. In C. Rauen (Hrsg.), Handbuch Coaching. Göttingen u. a.: Hogrefe.

Kühl, S. (2008). Coaching und Supervision. Personenorientierte Beratung in Organisationen. Wiesbaden: VS Verlag für Sozialwissenschaften.

Whitmore, J. (2009). Coaching für die Praxis. Wesentliches für jede Führungskraft. Frankfurt a. M.: Campus. 